\title{
Cholesterol Testing and Management: A National Comparison of Family Physicians, General Internists, and Cardiologists
}

\author{
Charles B. Eaton, MD, MS, Alicia Monroe, MD, William McQuade, MPH, MicabJ. Eimer
}

Background: We wanted to compare the frequency of cholesterol testing and treatment of hypercholesterolemia in patients cared for by family physicians, general internists, and cardiologists.

Methods: This study was a continuous cross-sectional survey of 1991 ambulatory office visits using a national probability sample of US physicians' office practices (National Ambulatory Care Survey). The physicians surveyed self-reported their specialty as family practice, internal medicine, or cardiology. Records of 33,795 patient visits to 1354 physicians were reviewed to find out whether the physicians reported cholesterol testing, cholesterol counseling, and charting of patient use of lipid-lowering medications. The results were compared among the three specialist groups.

Results: During an annual health examination ( 9.77 million office visits), a cholesterol test was reported by 23.5 percent of family physicians, 43.5 percent of internists, and 13.1 percent of cardiologists $(P<0.01)$. For all hypercholesterolemic patients (23.52 million office visits), the age- and sex-adjusted percentages of reported cholesterol-reduction counseling during office visits were 38.3 percent for family physicians, 42.4 percent for internists, and 36.5 percent by cardiologists (NS), and percentages of reported lipid-lowering medication prescriptions were 13.4 percent for family physicians, 25.1 percent for internists, and 28.4 percent for cardiologists $(P<0.01)$. In hypercholesterolemic patients with coronary heart disease ( 3.47 million office visits), the ageand sex-adjusted percentages of cholesterol reduction counseling reported during office visits were 64.4 percent for family physicians, 47.1 percent for internists, and 35.9 percent for cardiologists (NS) and the age- and sexadjusted percentages of lipid-lowering medication prescriptions reported were 13.9 percent for family physicians, 62.5 percent for internists, and 34.7 percent for cardiologists $(P<0.01)$.

Conclusions: Recommended goals regarding cholesterol testing and management were not reached by any physician group. Internists tested for hypercholesterolemia during an annual health examination more frequently and had more patients using lipid-lowering medications than did family physicians or cardiologists. Understanding the reasons for these specialty differences might lead to improvement in the diagnosis and management of hypercholesterolemia and therefore reduction in cardiovascular disease. (J Am Board Fam Pract 1998;11:180-6.

Cardiovascular disease is the leading cause of death and morbidity in the United States and most Western societies. ${ }^{1}$ Hypercholesterolemia is an important modifiable risk factor for cardiovascular disease. Appropriate detection, evaluation,

Submitted, revised, 29 May 1997.

From the Department of Family Medicine, Memorial Hospital of Rhode Island and Brown University School of Medicine, Pawtucket, Rhode Island. Address reprint requests to Charles B. Eaton, MD, MS, Memorial Hospital of Rhode Island, 111 Brewster Street, Pawtucket, RI 02860.

Supported in part by Grants Program for Research Externship funded by the American Academy of Family Physicians Foundation. Presented at North American Primary Care Research Group Meeting in Houston, Tex, in 1995. and treatment of hypercholesterolemia should lead to a marked reduction in cardiovascular disease death and morbidity. In 1988 the National Heart, Lung, and Blood Institute convened an expert panel that developed national guidelines for the detection, evaluation, and treatment of high blood cholesterol in adults - the National Cholesterol Education Program (NCEP). ${ }^{2}$ This expert panel recommended cholesterol screening every 5 years in asymptomatic patients and every 1 to 2 years in patients with cardiovascular disease or abnormal lipid profiles. All patients with hypercholesterolemia are recommended to be counseled regarding dietary changes, and if an explicit 
low-density lipoprotein (LDL) cholesterol goal is not reached, then lipid-lowering medication is recommended. For patients with established coronary heart disease, recommended thresholds to initiate lipid-lowering drug therapy are lower. Based upon the diet response in the Scandinavian Simvastatin Survival Study, ${ }^{3}$ an estimated 90 percent of patients with established coronary heart disease and hypercholesterolemia should be on lipid-lowering therapy.

Recent studies have suggested that hypercholesterolemia is underdetected and undertreated. ${ }^{4-10}$ A recent publication by Jollis et a ${ }^{11}$ purported to show that patients with acute myocardial infarctions cared for by cardiologists had better outcomes than patients cared for by family physicians and internists. In explaining these apparent differences in outcomes, Jollis et al ${ }^{11}$ speculated that differences in the frequency in the use of medications, such as aspirin, $\beta$-blockers, heparin, and nitroglycerin, could have explained the better outcomes attributed to cardiologists. We speculated the differences by specialty in the detection, evaluation, and treatment of hypercholesterolemia might be operative as well. Although descriptive studies describing the frequency of detection, counseling, and pharmacologic management of hypercholesterolemia by primary care physicians and cardiologists have been published, to our knowledge few, if any, between-specialty comparisons have been reported. $4,8-10$

By better understanding the similarities and differences in the detection, evaluation, and treatment of hypercholesterolemia between specialties, we can develop improved strategies to enhance risk-factor screening, counseling, and treatment of hypercholesterolemia for all physicians. The 1991 National Ambulatory Medical Care Survey (NAMCS) ${ }^{12}$ allows for a comparison of detection, counseling, and lipid-lowering medication utilization patterns with regard to hypercholesterolemia for representative samples of family physicians, internists, and cardiologists. Because this survey occurred 3 years after the release of the original NCEP guidelines, primary care physicians and cardiologists should have had enough time to have incorporated these guidelines into their practice.

\section{Methods}

NAMCS is a continuous survey of a nationwide probability sample of health care providers in the
United States administered by the National Center for Health Statistics. ${ }^{12}$ It attempts to capture objective information about ambulatory medical services provided in physicians' offices. Hospital clinics, urgent care centers, and community health centers are not included in the survey, and the services provided by independent nurse practitioners, chiropractors, and homeopaths are also not included. One hundred twelve counties or standard metropolitan statistical areas are sampled throughout the United States. Physicians representing 15 specialties from the American Medical Association and American Osteopathic Association master files are sampled from each area. The total physician sample is divided into 52 subsamples representing 1 week of patient visits.

The sampling rate for each office is based upon the size of the practice to insure complete data collection for each patient visit. The actual data collection is carried out by the physician and his or her office staff. Each physician records approximately 30 office visits per week. The nature of the patient's complaints, diagnosis, diagnostic or screening services provided, therapeutic services, medications, disposition, and time spent with the physician are recorded after each patient visit.

To provide unbiased national estimates, 33,795 records from 1354 physicians were abstracted and weighted according to standard cluster sampling techniques to estimate 669 million yearly office visits adjusting for sampling probabilities, nonresponse bias, and ratio adjustment by physician specialty. 12

Physician categories available were based upon self-report for family physicians, general practitioners, general internists, obstetrician-gynecologists, cardiologists, and cardiovascular surgeons. We chose to compare the hypercholesterolemia treatment practice patterns of family physicians with that of general internists and not include general practitioners, so that board certification requirements between primary care physicians would be similar. We did not evaluate the practice patterns of cardiovascular surgeons, since the sample was too small to make any meaningful inferences. To have representative samples of male and female patients, obstetrician-gynecologists were excluded.

Diagnoses were coded using the International Classification of Diseases, 9 th Revision, Clinical Modification (ICD-9-CM) ${ }^{13}$ ICD-9 codes 410 through 


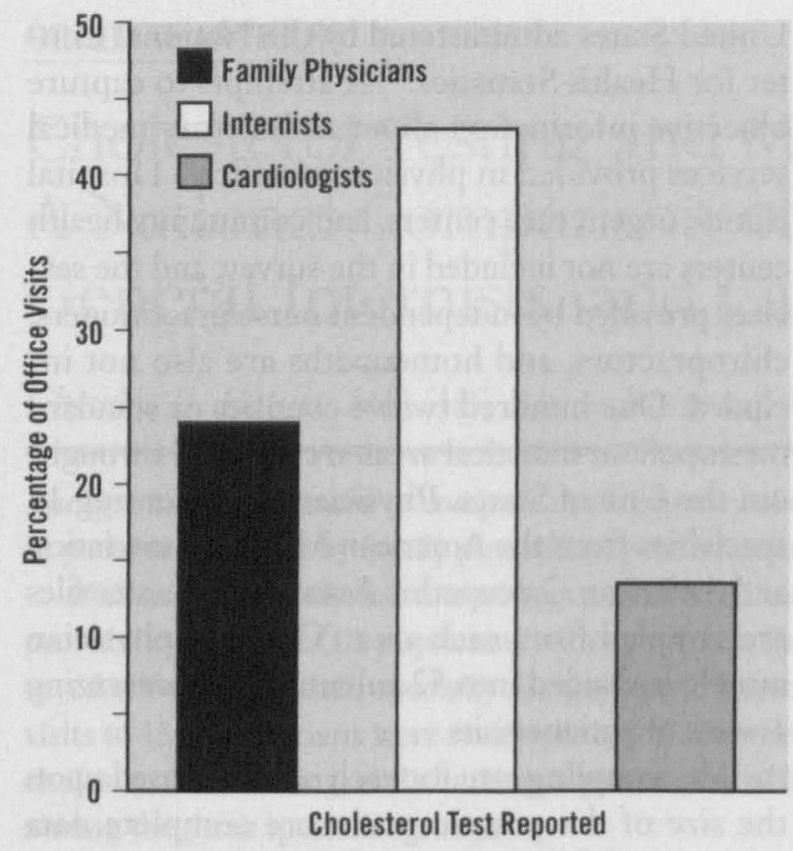

Figure 1. Percentage of annual health examination visits with cholesterol test performed. Data from the National Ambulatory Medical Care Survey, 1991. ${ }^{12}$

414 for any of three diagnoses coded per patient encounter were used to define coronary heart disease in this analysis. Reasons for visits were coded using A Reason for Visit Classification for Ambulatory Care developed by the American Medical Records Associations. ${ }^{14}$ An annual health examination was based upon physician reports using this code. Physician diagnostic and screening services, therapeutic services, and medications were assessed using the NAMCS data instrument. ${ }^{12}$ Physicians were asked to code after each visit whether the patient had hypercholesterolemia, whether a cholesterol test was ordered or performed, and whether counseling for hypercholesterolemia was performed. Use of lipid medications was ascertained from the list of medications recorded during each patient encounter. A specific question regarding a prescription for or patient utilization of specific lipid-lowering medications was not asked.

Statistical analysis was performed using SPSS. ${ }^{15}$ The frequency of cholesterol testing is given as a percentage; its calculation was based upon the weighted results determined by the cluster sampling used in the NAMCS survey. Analyses adjusted for age and sex differences in the patient populations between specialties used the weighted results and the three specialties' combined population as the standard population. Statistically sig- nificant differences between specialties in their frequency of cholesterol counseling and prescribing lipid-lowering medications were determined by performing a logistic regression on unweighted data, comparing internists with family physicians and cardiologists with family physicians, adjusting for age, sex, and where applicable, the diagnosis of coronary heart disease or annual health examination.

We used this conservative approach because the weighting procedures based upon cluster sampling are meant to be used only to estimate differences in the frequency for prespecified groups, such as physician specialties. Maximum likelihood estimating procedures used in logistic regression to adjust for multiple confounders are not valid when using the weighted results, because the standard errors associated with the weighted estimates for multiple strata of each variable are unknown.

\section{Results}

\section{Cholesterol Testing}

Of the 99 million estimated patient encounters by internists, 10 percent had cholesterol testing, whereas of the 48.6 million estimated patient encounters by family physicians, 6.1 percent had cholesterol testing. Cardiologists surveyed had an estimated 11.5 million patient encounters, of which 8.7 percent had cholesterol testing. Because the reason for visits might differ by specialty and, therefore, the appropriateness to perform cholesterol testing might differ, we compared rates of cholesterol testing during an annual health examination, when the indications for testing would be similar. The frequency of cholesterol testing was higher in all specialties during an annual health examination compared with all patient encounters. The differences between specialties for cholesterol testing during annual health examinations is displayed in Figure 1. Internists measured cholesterol levels in 43.5 percent, family physicians in 23.5 percent, and cardiologists in 13.1 percent of annual health examination encounters $(P<0.01)$.

Comparing the frequency of cholesterol testing for patients with established coronary heart disease regardless of the reason for the visit revealed no significant differences, with 15.8 percent of family physicians performing cholesterol testing, followed by 14.4 percent of internists, and then 9.1 percent of cardiologists.

The frequency of cholesterol testing varied 
Table 1. Percentage of Office Visits With Cholesterol Testing During Annual Health Examinations, 1991.

\begin{tabular}{lccccccr}
\hline & \multicolumn{2}{c}{ Family Physicians } & \multicolumn{2}{c}{ Internists } & \multicolumn{2}{c}{ Cardiologists } \\
Age, years & Male & Female & Male & Female & Male & Female & Total \\
\hline $18-29$ & 66.0 & 9.8 & 78.3 & 74.0 & - & - & 699,635 \\
$30-44$ & 75.1 & 29.2 & 59.3 & 42.6 & 100.0 & - & $1,554,861$ \\
$45-64$ & 25.7 & 15.4 & 22.7 & 63.4 & 25.2 & 4.0 & $3,168,702$ \\
$\geq 65$ & 19.9 & 10.8 & 1.7 & 35.6 & 19.3 & 13.9 & $4,355,489$ \\
Total & 38.6 & 15.5 & 37.6 & 46.7 & 16.1 & 9.4 & $9,778,687$ \\
\hline
\end{tabular}

From the National Ambulatory Medical Care Survey. ${ }^{12}$

significantly by age and sex of the patients, as shown in Table 1, which displays the age- and sex-specific cholesterol-testing frequency during annual health examinations for each specialty. Family physicians and cardiologists were more likely to measure cholesterol levels in men, whereas internists were more likely to measure cholesterol levels in women. This difference is striking for young women aged between 18 and 29 years, with 74 percent having cholesterol testing performed by internists and only 9.8 percent having cholesterol testing performed by family physicians. Family physicians and cardiologists were also less likely than internists to screen elderly patients (older than 65 years).

Adjusting for age, sex, and rates of annual physical examinations, internists were 83.8 percent more likely to perform cholesterol tests than family physicians $(\mathrm{OR}=1.83, \mathrm{P}<0.05)$, whereas cardiologists did not differ from family physicians in their rates of screening.

\section{Management of Hypercholesterolemia}

Figure 2 displays the age- and sex-adjusted rates of cholesterol reduction counseling and use of lipidlowering medications in patients with hypercholesterolemia, comparing family physicians with internists and cardiologists. There was no statistically significant difference in the frequency of cholesterol counseling during office visits between specialty groups for patients with hypercholesterolemia. Regarding the use of lipid-lowering medications, however, internists (25.1 percent) were much more likely than family physicians (13.4 percent) to have reported patients using lipid-lowering medications $(P<0.01)$. Cardiologists (28.4 percent) were also more likely to have reported patients using lipid-lowering medications than family physicians, but this result did not reach statistical significance because of the smaller number of patients seen by cardiologists.

Figure 3 displays the age- and sex-adjusted frequency of cholesterol reduction counseling and use of lipid-lowering medications in patients with hypercholesterolemia who also have a diagnosis of coronary heart disease, thus allowing for evaluation of secondary prevention cholesterol management practices. Here family physicians (61.9 percent) were more likely than internists (46.8 percent) or cardiologists (38.0 percent) to perform cholesterol reduction counseling during office patient visits, but these differences are not statistically significant. Regarding use of lipidlowering medications, internists (63.3 percent) were the most likely, followed by cardiologists (34.7 percent), and then family physicians (13.9 percent), to prescribe lipid-lowering medications to hypercholesterolemic patients with coronary heart disease $(P<0.01)$.

\section{Discussion}

The 1991 NAMCS survey provides a unique means to compare testing for and management of hypercholesterolemia by family physicians, internists, and cardiologists based upon a national probability sample. Our analysis suggests a significant interspecialty variation in the frequency of cholesterol testing and the frequency of drug therapy in the treatment of hypercholesterolemia. Internists were much more likely to order cholesterol testing during annual physical examinations (83 percent more likely than family physicians). Cholesterol testing is recommended every 5 years in asymptomatic persons and every 1 to 2 years in patients with cardiovascular disease or who have borderline or abnormal lipid levels according to the NCEP guidelines. ${ }^{3,16}$

The frequency of cholesterol testing found in 


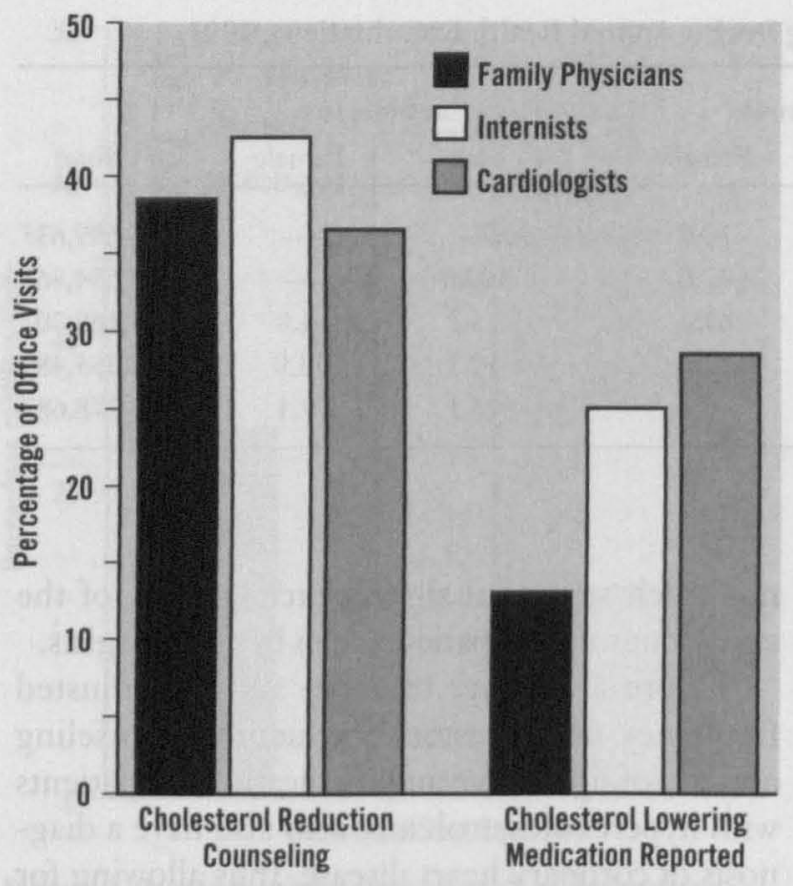

Figure 2. Age- and sex-adjusted prevalence of cholesterol treatment in patients with hypercholesterolemia. Data from the National Ambulatory Medical Care Survey, $1991 .{ }^{12}$

this cross-sectional survey needs to be interpreted within this longitudinal framework. Accordingly, it is difficult to comment on the appropriateness of cholesterol testing compared with national guidelines from the NAMCS data. The marked increase in testing noted by internists during an annual health examination might reflect a practice style of performing most preventive services at one time rather than interspersed within acute care visits, which might be more common in a family practice settings. Cardiologists might be seeing patients by referral and assuming that the primary care physicians will be performing cholesterol testing.

The marked sex differences in cholesterol testing during annual examinations shown in Table 1 (only 15 percent of women seen by family physicians compared with 46.7 percent seen by internists) deserves further investigation. These results could reflect competing demands, with family physicians focusing on gynecologic complaints or other women's health issues, but it does raise concerns about potential sex bias.

We found that with the exception of coronary heart disease patients cared for by family physicians, the frequency of cholesterol counseling for patients with diagnosed hypercholesterolemia was less than 50 percent. This frequency clearly reflects a less than optimal management of this important risk factor for coronary heart disease by all specialties.

We also found a low rate of pharmacologic therapy for patients with hypercholesterolemia and coronary heart disease. Family physicians reported prescribing lipid-lowering medications in less than 15 percent of patient encounters, compared with 62.5 percent for internists and 34.7 percent for cardiologists, after adjusting for age and sex. This finding suggests that significant specialty differences exist. The lack of an effective counseling or pharmacologic treatment plan for hypercholesterolemia by family physicians, internists, and cardiologists is consistent with reports in the medical literature..$^{4-10}$

Eaton et al, ${ }^{4}$ in a small study in one academic family medicine practice, found that for hypercholesterolemic patients with and without cardiovascular disease, between 72 and 74 percent had cholesterol testing, between 57 and 65 percent had dietary changes recommended, and between 20 and 26 percent were offered pharmacologic therapy, but only 2 to 7 percent had cholesterol levels controlled. Nieto et al, ${ }^{5}$ in the Atherosclerosis Risk in Communities study of 15,739 persons, showed that only 42 percent of hypercholesterolemic (total cholesterol levels $>240 \mathrm{mg} / \mathrm{dL}$ ) patients were aware of their diagnosis compared with 84 percent of hypertensive patients. Only 4 percent of hypercholesterolemic patients in this community study had their cholesterol treated and controlled. National Health and Nutrition Examination Survey III data collected from 1988 to $1991,{ }^{6}$ show that 87 percent of adults older than 20 years with fewer than two coronary heart disease risk factors have adequately controlled LDL cholesterol levels, whereas only 43 percent of adults with two or more risk factors have adequately controlled LDL cholesterol levels, and only 15 percent of adults with coronary heart disease have adequately controlled LDL cholesterol levels. Klein et al, ${ }^{7}$ in the Beaver Dam Eye Study, found that between 9.8 and 14.6 percent of hypercholesterolemic patients were on lipid-lowering therapy.

The differences by specialty in use of lipidlowering drugs for both primary and secondary prevention of coronary heart disease $(P<0.01)$, with family physicians prescribing medication less frequently, is not well documented in the medical 




Figure 3. Age-and sex-adjusted frequency of cholesterol treatment in patients with coronary heart disease. Data from the National Ambulatory Medical Care Survey, $1991 .{ }^{12}$

literature and is worthy of further investigation. These specialty differences could reflect a patientcentered negotiating style adopted by family physicians, in which lipid-lowering agents are not prescribed for patients unless they agree to be compliant; therefore, family physicians might counsel hypercholesterolemic patients more frequently. The differences in prescription patterns by specialty could represent confounding by health insurance status by specialty, as differences in the cost-benefit ratio for lipid-lowering therapy might vary for those patients who do not have a prescription plan covering the cost of lipid-lowering medications.

All the results of this study should be interpreted with caution. The data are self-reported and are prone to misclassification bias. We believe that such a bias would be nondifferential and therefore bias our results toward the null, making any interspecialty comparisons more difficult to uncover. Concerns about difference in the mix of patient diagnoses, age, sex, and severity of illness make interspecialty comparisons difficult to interpret. We attempted to deal with this issue by comparing patients with similar ICD-9 codes relevant to coronary heart disease and by comparing patients seen for a annual health examination. When comparing treatment frequency for hypercholesterolemia, we performed adjusted analyses to deal with confounding by age, sex of patients, and coronary heart disease. Other potential confounders, such as socioeconomic status, health insurance status, and physician age and sex, were not available for analysis.

Another potential weakness of this study is the time frame of the analysis. The NAMCS data were collected in 1991 and are 6 years old. This National Ambulatory Medical Care Survey is the first for which cholesterol testing and treatment data were collected. These data capture practice patterns of physicians 3 years after the release of the original NCEP guidelines. While the absolute percentages might be different in 1997 compared with 1991, it is hoped improved, specialty differences analyzed in this paper might well be stable. Future NAMCS data can be evaluated serially to test this hypothesis. The major strength of this study comes from its generalizability, as it estimates more than 669 million US physician office visits in 1991.

As confirmed by this study and others, ${ }^{4-10}$ hypercholesterolemia is underdetected and undertreated. It appears to be a universal problem for all specialties analyzed. Internists appeared to perform better than family physicians and cardiologists in the detection and drug treatment of hypercholesterolemia in this study. Future research to better understand these differences might be helpful for changing physician practice patterns so that the primary and secondary prevention of coronary heart disease can be optimized by devising better methods for the detection and management of hypercholesterolemia. More effective use of practice guidelines, office-based systems, pharmacists, nurses, physician extenders, or medical informatics might be needed to reach national goals of optimal cholesterol management.

\section{References}

1. National Heart, Lung, and Blood Institute report of the task force on research in epidemiology and prevention of cardiovascular disease. Washington, DC: US Department of Health and Human Services, Public Health Service, National Institutes of Health, 1994:ix-xiv.

2. Report of the National Cholesterol Education Program Expert Panel on Detection, Evaluation, and Treatment of High Blood Cholesterol in Adults. The Expert Panel. Arch Intern Med 1988;148:36-69. 
3. Randomized trial of cholesterol lowering in 4444 patients with coronary heart disease: the Scandinavian Simvastatin Survival Study (4S). Lancet 1994; 344:1383-9.

4. Eaton CB, McQuade W, Glupczynski D. A comparison of primary versus secondary cardiovascular disease prevention in an academic family practice. Fam Med 1994;26:587-92.

5. Nieto FJ, Alonso J, Chambless LE, Zhong $M$, Ceraso M, Romm FJ, et al. Population awareness and control of hypertension and hypercholesterolemia. The Atherosclerosis Risk in Communities study. Arch Intern Med 1995;155:677-84.

6. Sempos CT, Cleeman JI, Carol MD, Johnson CL, Bachorik PS, Gordon DJ, et al. Prevalence of high blood cholesterol among US adults. An update based on guidelines from the second report of the National Cholesterol Education Program Adult Treatment Panel. JAMA 1993;269:3009-14.

7. Klein R, Klein BE, Wang Q, Jensen SC. Treatment and control of hypercholesterolemia and hypertension in persons with and without diabetes. Am J Prev Med 1995;11:329-35.

8. Scott CS, Leaf D, Neighbor WE, Schaad DC, Brock DM, Van Citters RL. Preventive cardiology education and practice in residency training: residents' attitudes, perceptions, and practices. Am J Prev Med 1990;6:(2 Suppl):60-9.

9. Lewis $C$, Jenkins $P$, Pearson TA. Detection and management of high blood cholesterol: what providers think vs. what providers do. Circulation 1992;85:870.
10. Elmer PJ, Trenkner L, Finnegan J, Luepker RV, Laing B, Eszler J. Evaluation of cardiovascular disease (CVD) risk factor management in rural primary care practice. Circulation 1992;85:870.

11. Jollis JG, DeLong ER, Peterson ED, Muhlbaier LH, Fortin DF, Califf RM, et al. Outcome of acute myocardial infarction according to the specialty of the admitting physician. N Engl J Med 1996;335:1880-7.

12. National ambulatory medical care survey: 1991 summary. Advance data from vital and health staristics, no. 230. Hyattsville, Md: National Center for Health Statistics, 1991. (DHHS publication no. [PHS] 91-1250.)

13. International classification of diseases, 9 th revision, clinical modification. 2nd ed. Washington, DC: Public Health Service, Health Care Financing Administration, 1980.

14. Schneider D, Appleton L, McLemore T. A reason for visit classification for ambulatory care. National Center for Health Statistics. Vital Health Stat 1979; 2(78):i-vi, 1-63.

15. SPSS 6.1 [computer program]. Chicago: SPSS Inc, 1994.

16. Second report of the expert panel on detection, evaluation, and treatment of high blood cholesterol in adults (adult treatment panel II). Bethesda, $M d$ : National Cholesterol Education Program, National Institutes of Health, National Heart, Lung, and Blood Institute, 1993. (NIH publication no. 9330956.) 